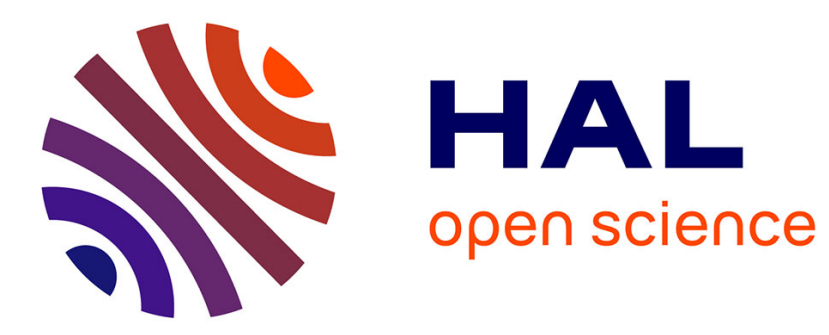

\title{
LINKS BETWEEN THE HARTREE-FOCK AND SEMICLASSICAL MODELS OF STATIC NUCLEAR PROPERTIES
}

\author{
F. Tondeur, J. Arcoragi, Justin Pearson
}

\section{- To cite this version:}

F. Tondeur, J. Arcoragi, Justin Pearson. LINKS BETWEEN THE HARTREE-FOCK AND SEMICLASSICAL MODELS OF STATIC NUCLEAR PROPERTIES. Journal de Physique Colloques, 1984, 45 (C6), pp.C6-125-C6-135. 10.1051/jphyscol:1984615 . jpa-00224216

\section{HAL Id: jpa-00224216 https://hal.science/jpa-00224216}

Submitted on 1 Jan 1984

HAL is a multi-disciplinary open access archive for the deposit and dissemination of scientific research documents, whether they are published or not. The documents may come from teaching and research institutions in France or abroad, or from public or private research centers.
L'archive ouverte pluridisciplinaire HAL, est destinée au dépôt et à la diffusion de documents scientifiques de niveau recherche, publiés ou non, émanant des établissements d'enseignement et de recherche français ou étrangers, des laboratoires publics ou privés. 


\title{
LINKS BETWEEN THE HARTREE-FOCK AND SEMICLASSICAL MODELS OF STATIC NUCLEAR PROPERTIES
}

\author{
F. Tondeur, J.P. Arcorag $i^{\star}$ and J.M. Pearson ${ }^{\star}$ \\ Universite Libre de Bruxelzes, CP 229, B 1050 Bruxelles, Belgium \\ and \\ Institut Supérieur Industriel de Bruxelzes, Belgium \\ * Département de Physique, Université de Montréal, Montréal, Canada
}

\begin{abstract}
Résumé - I'équivalence entre le modèle de Hartree-Fock (HF) et la méthode de Thomas-Fermi étendue (ETF) avec corrections de couches est examinée. Une prescription de strutinsky gếnéralisée est développée pour des spectres finis. Sa bonne précision est démontrée dans le cadre de l'approche selfconsistente Hartree-Fock-Strutinsky (HFS). Les résultats de calculs HFS et ETF sont comparés pour un grand nombre de noyaux, montrant un accord global acceptable mais aussi certains désaccords systématiques. Le choix de l'interaction effective dans les deux modèles est enfin discuté. En utilisant un ensemble de forces de skyrme développé récemment et qui permet une approche systématique de la question, plusieurs contraintes sont suggérées pour ajuster une force donnant de bonnes extrapolations.
\end{abstract}

\begin{abstract}
The equivalence between the Hartree-Fock (HF) model and the extended Thomas-Fermi (ETF) method with shell corrections is examined. A generalised strutinsky prescription is developed for finite spectra. Its good accuracy is shown in the framework of the self-consistent HartreeFock-Strutinsky (HFS) approach. Then, HFS and ETF results are compared for a large number of nuclei, showing an acceptable overall agreement but also several systematic discrepancies. The choice of the effective interaction for both models is finally discussed. By using a recently developed set of Skyrme forces which allows a systematic approach of this question, several constraints are suggested for the fit of a force suitable for extrapolations.
\end{abstract}

\section{1) Introduction}

The past ten years have seen the extraordinary development of the use of Skyrme forces in Hartree-Fock [1] and semi classical nucleax models [2]. The popularity of these forces is due to their ability to reproduce many static nuclear properties with a good accuracy [3]. Among many other results obtained with those forces, we would like to point out the demonstration of the deficiencies of more phenomenological models, in particular the droplet model [4]. It has thus become evident that Hartree-Fock (HF) or semi classical models should be used systematically instead of the droplet model (DM), especially in all cases where no experimental result is available to check the accuracy of the model, e.g. for extrapolations far from stability.

The HF model should in principle be more accurate than semi classical approximations like the extended Thomas-Fermi model (ETF). Unfortunately, its application to deformed nuclear shapes [5] implies heavy numerical calculations, and cannot be used for systematic studies of several thousand nuclei, as needed for example in astrophysical applications. Thus we have still to rely on an approximate method for deformed shapes, like the ETF + shell correction method which improves the usual "macroscopic-microscopic" calculations by using ETF instead of the DM for the macroscopic part.

In ETF with Skyrme forces, the binding energy of the nucleus can be calculated 
from the density distributions $P_{n}$ and $P_{p}$ by using the semi classical expansion of the kinetic and spin-orbit densities [6] . Brack and coworkers minimize it by using generalized Fermi trial distributions given here for the spherical case :

$$
\rho_{q}(r)=\rho_{0 q}\left[1+\exp \left(\frac{r-R}{a}\right)\right]^{-\gamma}
$$

where $q=n$ or $p$. When $\rho_{n}$ and $\rho_{p}$ have been determined, the corresponding singleparticle potential $V_{\text {ETF }}$ can be easily obtained.

After calculating the single particle levels $e_{i}$ in $V_{E T F}$, one can determine the shell correction to the energy :

$$
j_{1} B=\sum_{\text {occ. }} e_{i}-\left\langle\sum e_{i}\right\rangle_{\text {average }}
$$

which added to $B_{E T F}$ is expected to give a reasonable approximation to $\mathrm{HF}$ :

$$
B_{H F} \stackrel{?}{\approx} B_{E T F}+S_{1} B
$$

Two methods can be used to evaluate the average sum of single-particle energies in eq. (2) : the strutinsky prescription [7] or the semiclassical method [8]. The standard strutinsky method meets some problems when applied to finite spectra and can be difficult to operate for occasional users who do not have the "know-how". The semiclassical method being easier to handle and completely unambiguous can thus be prefered. However, the Strutinsky method also allows for a self-consistent calculation of the macroscopic energy [9] without making use of the semiclassical approximation. We shall refer to this type of calculation as to the Hartree-Fock Strutinsky (HFS) method. The HFS method is as costly as HF, and thus not of interest in itself, but can be used to check the accuracy of the semiclassical models ( $x$ of the $D M$ ) and in particular the accuracy of the ETF density distributions, single-particle potentials and shell corrections.

We shall show in section 2 that it is possible to overcome the problem met with the Strutinsky prescription for finite spectra. We indeed present a generalised smoothing prescription which can be used in this case. We compare in section 3 several HFS results obtained with this new prescription to the corresponding ETF results. Finally, section 4 examines a common problem to all methods : the choice of the effective skyrme interaction.

\section{2 - Shell corrections method for finite spectra}

The standard strutinsky prescription [7] can only be used if at least three major shells are known above the Fermi energy in the single-particle spectrum. In realistic potentials, only one shell is usually available and two shells of positive-energy levels must be defined according to more or less empirical recipes [10]. A technical discussion of the results given in [11] has shown the feasability of the method in the favourable case of $\mathrm{N}=\mathrm{Z}$ nuclei without any coulomb force, for which the bound spectrum includes moxe than one empty shell and sometimes two. The method however is not easy to handle for occasionnal users and presents serious difficulties when applied near the neutron drip line.

Two approaches have been followed to improve the prescription for finite spectra. One has been developed by Ivanjuk and Strutinsky [12], but has not been applied to systematic calculations. The second one is described here, and improves the method developed in a previous work [13].

Starting with the"occupation number representation" (with $q=n$ or $p$ ) : 


$$
\tilde{E}_{q}=\sum_{a l l} \tilde{n}_{i q} e_{i q}
$$

we introduce the following constraints on $\tilde{n}_{\text {iq }}$ :

$$
\sum_{\text {all }} \tilde{n}_{i}\left(i^{1 / 3}\right)^{k}=\sum_{\text {occ }}\left(i^{1 / 3}\right)^{k}, k=0,4
$$

which express that the averaging made in eq. (4) should not modify $E=\sum_{\text {oce }} e_{i q}$ if $\boldsymbol{e}_{\text {iq }}$ is already a smooth function of $i$. (All states are supposed to be nondegenerate and ordered by increasing energy). The variable $i^{1 / 3}$ is chosen in view of the average trend $e_{i} \sim i^{1 / 3}$ valid for equidistant shells. Eq. (5) also includes the nucleon number conservation for $\mathrm{k}=0$. A parametrised function is chosen for $\widetilde{n}_{i}$ :

$$
\tilde{n}_{i q}=\frac{a_{0 q}}{1+\exp \left(\left(i^{1 / 3}-N_{q}^{1 / 3}\right) / \gamma\right)}+\frac{\sum_{l} a_{l q}\left(i^{1 / 3}\right)^{l-1}}{\cosh ^{2}\left(\left(i^{1 / 3}-N_{q}^{1 / 3}\right) / 2 \gamma\right)}
$$

where the $\boldsymbol{a}_{\boldsymbol{l}}$ are determined by eq. (5), $\boldsymbol{N}$ being the number of nucleus of type $q$. With $i^{1 / 3}$ taken as the variable, the value of the smoothing parameter $\gamma$ which corresponds to one shell spacing is roughly the same in the whole $(\mathbb{N}, z)$ plane. In the standard prescription, $\gamma$ is chosen to fulfil the plateau condition :

$$
d \tilde{E} / d Y=0
$$

However, as discussed in [13], this condition is not necessary. We instead ask a good accuracy of the Strutinsky theorem with a smooth $\widetilde{B}$ and the usual shell correction :

$$
B_{H F} \approx \tilde{B}+\sum_{\text {occ. }} e_{i}-\sum_{a l l} \tilde{n}_{i} e_{i}
$$

As demonstrated in $[9,13]$ and hereafter, the theorem is accurate if $\widetilde{B}$ is calculated self-consistently by replacing in HF the HF occupation numbers by the $\tilde{n}_{i}$. However, $\widetilde{B}$ HFS is not necessarily a smooth function [13]. To get a smooth $\widetilde{B}_{\mathrm{HFS}}$, it is necessary :

a) to avoid the brutal changes in the level order and in the $\tilde{n}_{i}$ when highly degenerate levels cross each other in the $(N, Z)$ plane, this can be done by introducing a fictive smooth level order.

b) to avoid the brutal variation of the total number of states in the spectrum by including a smooth number of states $\boldsymbol{n}(\mathrm{N}, 2)$ which is usually different from the total number of bound states.

c) to smooth the irregular effects introduced by the shell fluctuations of the upper limit of the considered spectrum, by calculating the $\tilde{n}_{i}$ for a few different values of $\boldsymbol{n}$ and making an average.

d) to damp explicitely in the smoothing prescription the oscillating component of $e_{i}$; with the variable $i^{1 / 3}$, this component is roughly periodical (with the period $\frac{2}{3}$ ) and its lower harmonic can be cancelled in $\widetilde{E}$ by introducing two new constraints:

$$
\sum_{\text {all }} \tilde{n}_{i} \sin \left(3 \pi i^{1 / 3}\right)=\sum_{\text {all }} \tilde{n}_{i} \cos \left(3 \pi i^{1 / 3}\right)=0
$$

With those improvements, $\tilde{B}(N, Z)$ is found to be smooth within $\pm I \mathrm{MeV}$ for nuclei along the stability line. 
The additional constraints (9) do not affect too much the accuracy of the strutinsky theorem, except in a few cases where the 7 constraints $(5,9)$ are probably too strong and lead to pathological results, like occupation numbers much higher than 1.5. Varying $Y$ usually solves the problem. As a general rule, the 7 constraints leave only a very small freedom. In particular, the $\widetilde{n}_{i}$ are finally quite unsensitive to the value of $\gamma$ even when this paraneter changes by an order of magnitude (The plateau condition thus becomes meaningless). It is also very difficult to add more constraints (e.g. the damping of higher harmonics of the oscillating part of $\left.e_{i}\right)$.

The higher order shell correction

$$
S_{2} B=B_{H F}-\tilde{B}_{H F S}-\delta_{1} B
$$

is found in most case within the range $\delta_{3} B=0.75 \pm 0.50 \mathrm{MeV}$. The largest values are obtained for magic nuclei, for which $J_{,} B$ is also larger. As $J_{Z} B$ is always positive, the shell correction can be improved by including its average value, which does not vary significantly with $A$ :

$$
B_{H F} \approx \widetilde{B}_{H F S}+0.75-\delta, B
$$

the average error being then lower than $0.25 \mathrm{MeV}$. Such a good accuracy is also found when pairing correlations axe taken into account :

$$
B_{H F B C S} \approx \tilde{B}_{H F S}+0.75+\sum\left(v_{i}^{2}-\tilde{n}_{i}\right) e_{i}-\frac{\Delta_{n}^{2}}{G_{n}}-\frac{\Delta_{p}^{2}}{G_{p}}
$$

except in a few cases where the BCS occupation numbers $v_{i}^{2}$ calculated for the HF'S single particle states are very different from those obtained for the HFBCS spectrum. This problem mostly concerns a few light or medium nuclei, and is likely to occur also in models where $B_{\text {HFS }}$ and the mean field $v_{\text {HFS }}$ are replaced by their semiclassical estimates.

\section{3 - Comparison between extended Thomas-Fermi and Hartree-Fock Strutinsky results}

The substitution of semiclassical quantities to the smooth HFs binding energies and single-particle potnetials however introduces much larger uncertainties, as will now be shown. The comparison between $V_{\text {ETF }}$ and $V_{H F S}$ will be done elsewhere. We shall focuse the present discussion on a comparison between density distributions and between binding energies.

The ETF model used here incIudes the fourth order expansion of the kinetic density [6] but only retains the second order terms for the spin-orbit terms (including the spin-orbit contribution to the kinetic energy). The generalised Fermi shapes of eq. (1) are used as trial wavefunctions, but the constraint $\gamma=1$ has been imposed in some cases. All calculations use the skyrme force T1 [14, 15]. We compare in fig. 1 the ETF and HFS results for the bulk density of neutrons and protons in nuclei near the stability line. In the HFS case, the bulk density is obtained as in [16] by fitting a Fermi distribution to the HFs distributions. When $Y \neq 1$ is allowed, the agreement between the two models is quite good, especially for heavy nuclei. In light and medium nuclei, the ETF densities are a bit too low, but the disagreement does not exceed $3 \%$.

Fig. 2 compares the $90 \%$ - $10 \%$ diffusenesses obtained with $\gamma=1$, showing the usual underestimate of this quantity by the ETF model. The agreement with HFS is improved with $Y \neq 1$, but the ETF surface diffuseness is still too small on the average. Moreover, the increase of $t_{90-10}$ with $\gamma^{\gamma} \neq 1$ is mainly located inside of 


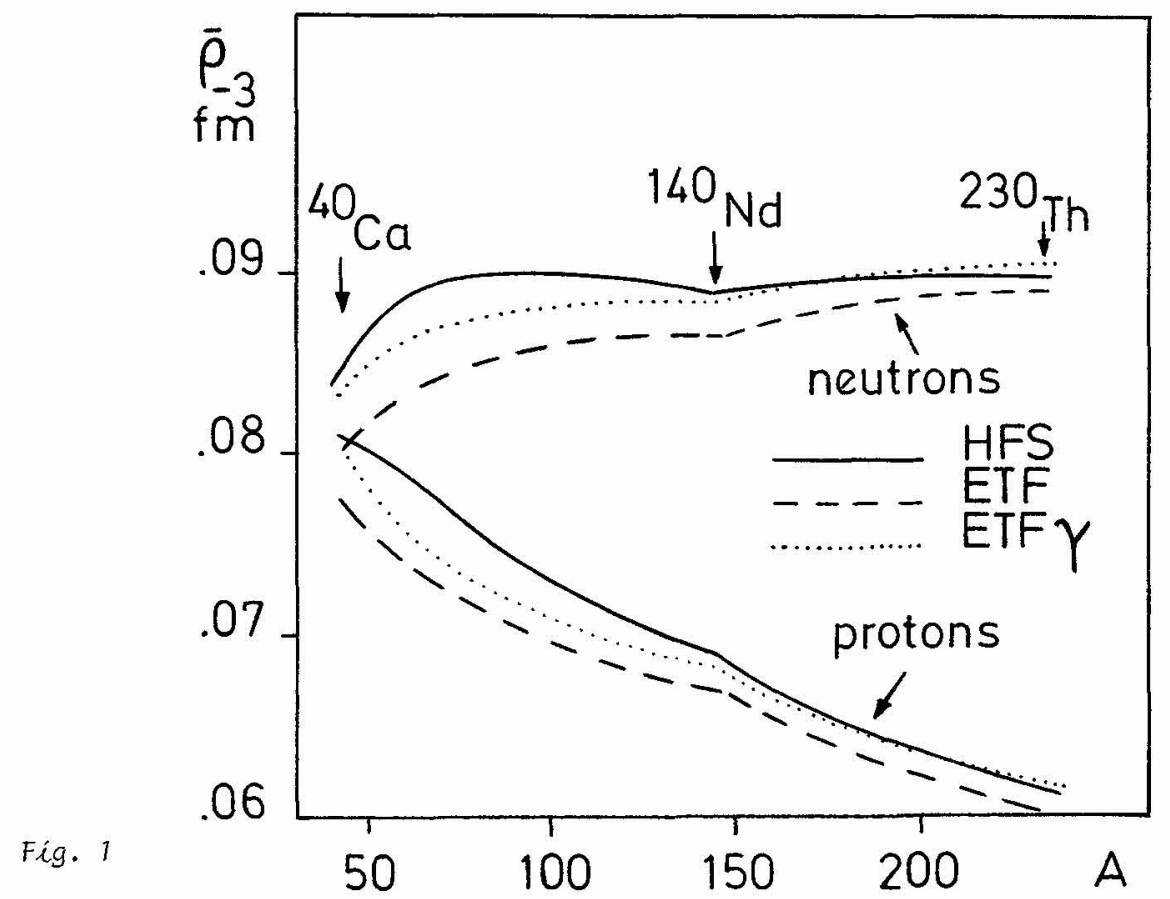

Comparison of the core densities in HFS and ETF models. ETFY refers to ETF with generalised Fermi shapes $(\boldsymbol{\gamma} \neq 1)$

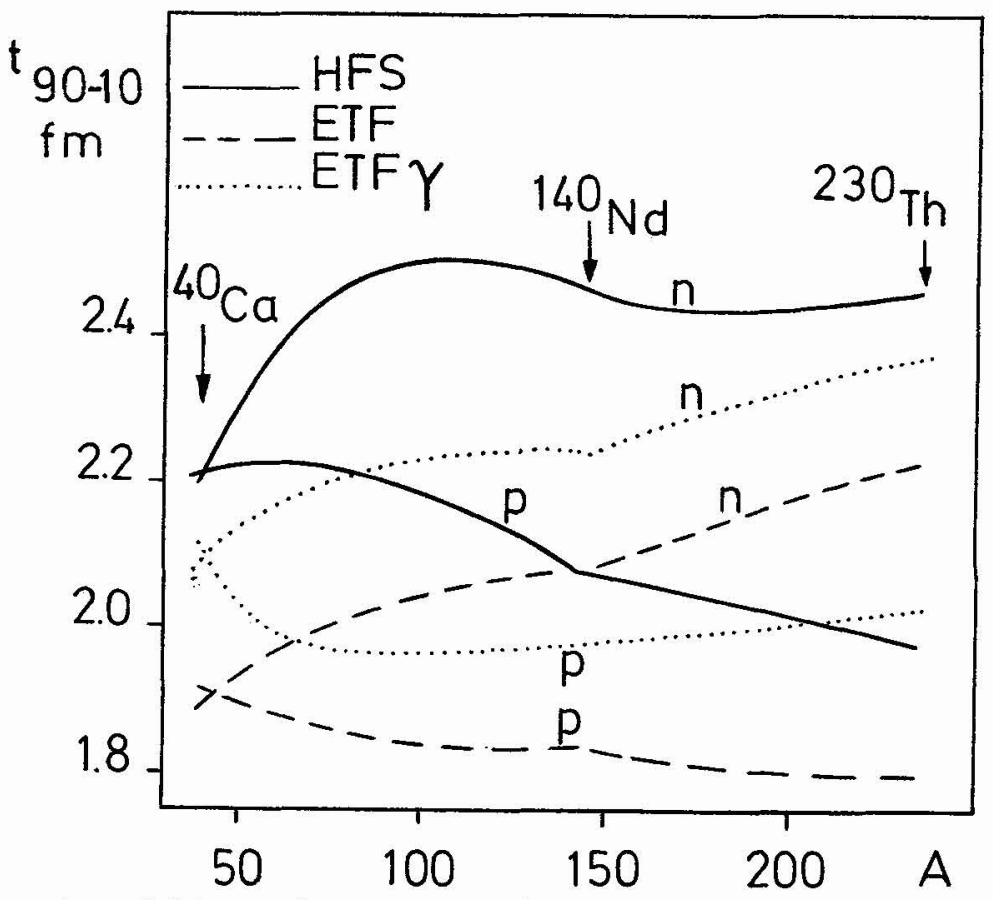

Fig. 2

$90 \%$ - 10\% surface thickness for neutron and proton distributions in HFS and ETF models 
the surface, whereas HFS results predict a largex diffuseness for the outex part of the surface (i.e. the 50\% - 10\% compared with the $90 \%$ - 50\% diffuseness). We also note the different trends obtained for the proton diffuseness in HFS and ETF with $\gamma \neq 1$. Fig. 3 compares the values of $\gamma$ obtained by the ETF model and by a fit to HFS distributions. The most striking result is in the completely different trends for $\gamma_{p}$. On the other hand, the trends of $\gamma_{n}$ are quite similar, but the values differ by $\approx 30 \%$.

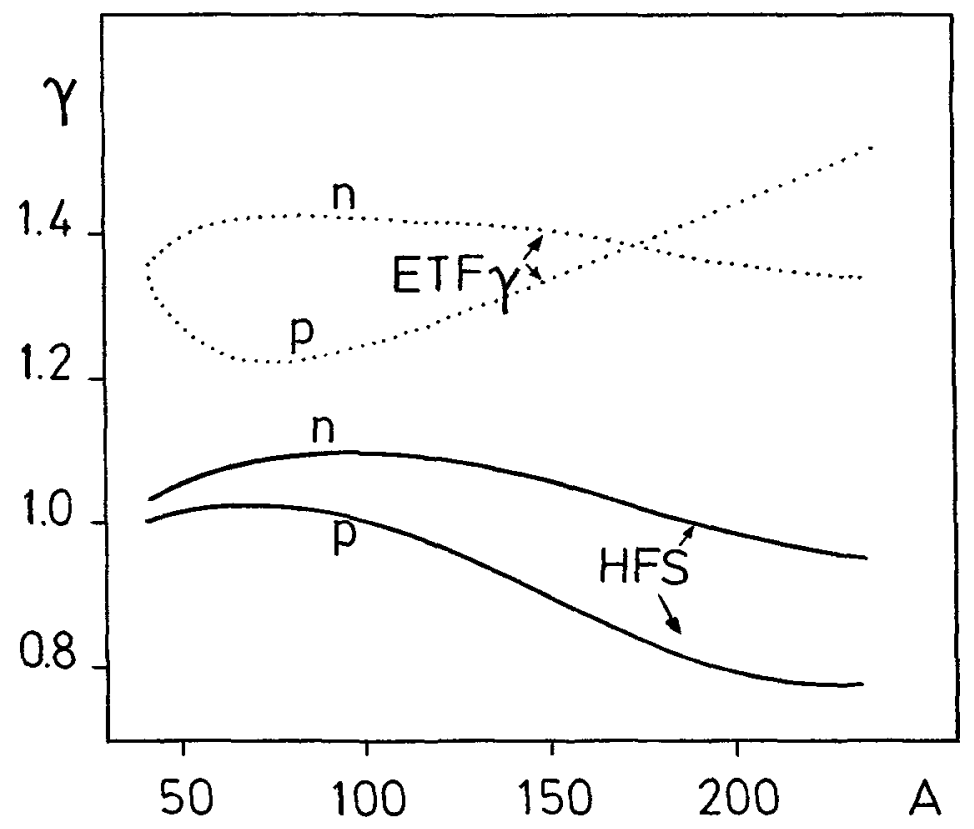

Fig. 3 - Surface asymmetry parameter $\gamma$ of the generalised Fermi distribution for neutrons and protons in HFS and ETF models; $\gamma=1$ corresponds to the pure Fermi shape with a symmetric surface.

\section{Table 1}

Hartree-Fock-Strutinsky (HFS) results compared with extended-Thomas-Fermi results with pure Fermi density distributions (ETF) or with generalized Fermi shapes given by eq. (1) (ETFY) for the different contributions to the binding energies of $200_{\mathrm{Hg}}(\mathrm{in} \mathrm{MeV}\rangle$.

HFS

kinetic

central

Coulomb

surface

spin-orbit

total

$$
\begin{array}{r}
\multicolumn{1}{c}{\text { कTF }} \\
3760.4 \\
-6266.9 \\
766.4 \\
259.8 \\
-87.9 \\
-1568.3
\end{array}
$$$$
\text { ETF }
$$$$
3769.7
$$$$
-6273.4
$$$$
768.4
$$$$
247.7
$$$$
-82.9
$$$$
-1570.5
$$ 
These disagreements between HFS and ETF descriptions of the surface significantly limit the accuracy one can expect to obtain in ETF calculations if the same force is used as in HF. Indeed the validity of the HF approach is fully confirmed by the excellent fits to experimental charge densities which can be achieved. In particular, that $y_{p}<1$ in heavy nuclei is clearly indicated by the experimental charge distributions in ${ }^{208} \mathrm{~Pb}$. In view of the key-role played by the density distributions in ETF, one can also expect significant inaccuracies in other quantities obtained in this model.

We are particularly interested in the binding energies. We present in Table 1 the results obtained for $200 \mathrm{Hg}$. It must be noted that the inclusion of fourthorder spin-orbit terms would improve the agreement between HFS and ETF : according to Bxack's results [2], these corrections reduce the spin-orbit energy by $\sim 208$ in this region. However, differences amounting to several MeV are found between the two models, in particular in the $(\nabla \rho)^{2}$ terms of the energy density functional, which reflect the differences noticed in the surface diffusenesses. We cannot expect that the approximation of $\widetilde{B}_{\mathrm{HFS}}$ by $\mathrm{B}_{\mathrm{ETF}}$ will be as accurate as the strutinsky method. Thus, in order to obtain a satisfactory approximation to HF, it is necessary to introduce small empirical corrections to the ETF method. We can imagine to do this at three different levels :

a) the skyrme force can be refitted. This is certainly the most reasonable choice if the ETE + shell correction method is used for itself, with no intention to give an approximation to $\mathrm{HF}$, the force being then fitted in order to reproduce experimental results with the ETF + shell correction method. Preliminary results show that the HF interaction must only be slightly modified, at least when the semiclassical shell corrections are used [8]. Work in this direction is currently under way, and will be reported elsewhere;

b) the ETF expansion of the kinetic and spin-orbit densities can be slightly modified by including empirical terms. This would give a natural unification of the MTF (zeroth order TF + empirical terms) and ETF (fourth order ETF without empirical terms) approaches;

c) empirical corrections can be introduced in the results themselves, e.g. the binding energies, by comparing ETF and HFS in a few selected cases, the corrections being interpolated for other cases. It would however be completely equivalent to interpolate directly the HFS results without using ETF, as done in [17].

\section{The choice of the effective interaction}

As discussed above, the HF and ETF effective forces can be slightly different. However, only minor differences are expected, and both interactions must fulfil the same general requirements. In particular, it is usually asked to reproduce experimental binding energies and charge radii with a sufficient accuracy. If we want to use HF and ETF in the whole field covered by the DM, we must also add a good fit to fission barriers. None of the published versions of the Skyrme forces simultaneously fulfils all these constraints. Most of them fail to reproduce fission barrier heights, whereas other (like SkM * [18] ) do not fit the binding energy of neutron-rich nuclei. Recently, we have developed a set of trial forces

[15], which allow for a systematic approach to the interplay between the parameters of the forces and the ETF/HF results, by allowing for separate variations of different physical parameters. Definitive fits have not yet been made. We can however summarize a few results already obtained with these forces, the parameters of which are given in Table 2. All forces are fitted to a few binding energies $\left(16_{0}, 56_{\mathrm{Fe}}, 90_{\mathrm{Zr}}, 118 \mathrm{Sn}, 132 \mathrm{Sn}, 138_{\mathrm{Ba}}, 146_{\mathrm{Gd}}, 208_{\mathrm{Pb}}\right)$ and to the charge radius of $208_{\mathrm{Pb}}$.

The way they are derived from each other is schematically given in fig. 4. In this figure, 3 stands for the coefficient of thel $\left.\nabla\left(\rho_{n}-\rho_{p}\right)\right|^{2}$ term in the skyrme Hamiltonian density :

$$
3=-\frac{1}{64}\left(3 t_{1}+t_{2}+6 t_{1} x_{1}+2 t_{2} x_{2}\right)
$$




\section{Table 2}

Coefficients of Skyrme forces and corresponding Droplet Model parametexs. The values of $\mathrm{m}^{*} / \mathrm{m}$ are given for symmetric nuclear matter.

\begin{tabular}{|c|c|c|c|c|c|c|c|c|c|}
\hline & TI & $\mathrm{T} 2$ & $\mathrm{~T} 3$ & $\mathrm{~T} 4$ & T5 & $\mathrm{T} 6$ & T7 & T8 & $\mathrm{pg}$ \\
\hline$t_{0}$ & -1794.0 & -1791.6 & -1791.8 & -1808.8 & $-2917.1-$ & -2794.2 & -1892.5 & -1892.5 & -1891.4 \\
\hline$t_{1}$ & 298 & 300 & 298.5 & 303.4 & 328.2 & 294 & 366.6 & 367 & 377.4 \\
\hline$t_{2}$ & -298 & -300 & -99.5 & -303.4 & -328.2 & -294 & -21 & -228.76 & -239.16 \\
\hline$t_{3}$ & 12812 & 12792 & 12794 & 12980 & 18584 & 12817 & 11983 & 11983 & 11982 \\
\hline$x_{0}$ & .154 & .154 & .138 & -.177 & -.295 & .392 & .334 & .448 & .441 \\
\hline$x_{1}$ & -.5 & -.5 & -1 & -.5 & -.5 & -.5 & -.359 & -.5 & -.5 \\
\hline$x_{2}$ & -.5 & -.5 & 1 & -.5 & -.5 & -.5 & 6.9 & -.5 & -.5 \\
\hline$x_{3}$ & .089 & .089 & .075 & -.5 & -.5 & +.5 & .366 & .695 & .686 \\
\hline$\alpha$ & $1 / 3$ & $1 / 3$ & $1 / 3$ & $1 / 3$ & $1 / 6$ & $1 / 3$ & .285 & .285 & .285 \\
\hline$w_{0}$ & 110 & 120 & 126 & 113 & 114 & 107 & 109 & 109 & 130 \\
\hline $\mathrm{k}_{\mathrm{F}}$ & 1.336 & 1.336 & 1.336 & 1.330 & 1.344 & 1.336 & 1.335 & 1.335 & 1.334 \\
\hline$P_{\infty}$ & .1610 & .1610 & .1611 & .1590 & .1640 & .1609 & .1606 & .1607 & .1608 \\
\hline $\mathrm{a}_{\mathrm{V}}$ & -15.98 & -15.94 & -15.96 & -15.95 & -15.99 & -15.96 & -15.94 & -15.94 & -15.88 \\
\hline K & 236.1 & 235.7 & 235.9 & 235.5 & 201.7 & 235.9 & 235.6 & 235.7 & 234.9 \\
\hline J & 32.02 & 32.00 & 31.50 & 35.45 & 37.00 & 29.97 & 29.52 & 29.92 & 29.76 \\
\hline L & 56.2 & 56.2 & 55.3 & 94.1 & 98.5 & 30.9 & 31.1 & 33.7 & 33.7 \\
\hline$a_{S} \mathrm{HF}$ & 18.3 & 18.1 & 17.9 & 18.2 & 18.2 & 18.3 & 18.3 & 18.2 & 17.9 \\
\hline$a_{s}$ ETF & 17.99 & 17.77 & 17.66 & 17.90 & 17.97 & 17.95 & 17.90 & 17.90 & 17.61 \\
\hline$Q^{H F F}$ & 33. & 33. & 34. & 26. & 25 . & 40 & 41. & 41 . & 43 \\
\hline $\mathrm{Q}^{\mathrm{ETF}}$ & 36.5 & 36.4 & 38.5 & 27.9 & 26.9 & 46.8 & 46.7 & 46.1 & 45.1 \\
\hline$\frac{\mathrm{m}^{*}}{m}$ & 1 & 1 & $I$ & 1 & 1 & 1 & $5 / 6$ & $5 / 6$ & $5 / 6$ \\
\hline
\end{tabular}

whereas the isoscalar and isovector $\left(\mathrm{m}^{*}\right)^{-1}$ refer to the two contributions to $\mathrm{m}^{*-1}$ :

$$
m_{q}^{*-1}=m_{q}^{-1}+\frac{\rho}{8 \hbar^{2}}\left(3 t_{1}+5 t_{2}+4 t_{2} x_{2}\right) \pm \frac{\rho_{n}-\rho_{b}}{8 t^{2}}\left(t_{2}-t_{1}+2 t_{2} x_{2}-2 t_{1} x_{1}\right)
$$

where $q=n$ or $p$, the + sign holding for neutrons and the - sign for protons. The notation $x_{3}\left(x_{0}\right)$ is used to indicate correlated variations which are constrained by the fit to the masses. As usually, $K$ is the nuclear matter compression modulus and $w_{0}$ the spin-orbit strength.

a) the fit of HF results to the experimental binding energies is better when $\mathrm{m}^{*} \approx \mathrm{m}$. For a reasonable $\mathrm{m}^{*}\left(\approx \frac{2}{3} \mathrm{~m}\right.$ to $\left.\mathrm{m}\right)$, this fit allows the determination of at least three parameters, which $\overline{3}$ correspond roughly to the volume, surface and asymmetry contributions of the droplet model, the coulomb energy being fixed by the fit to the radius of $208 \mathrm{~Pb}$. The volume and surface coefficients only slightly depend on the force (Table 2). The surface energies are determined here by HF or EnF calculations for semi-infinite nuclear matter. Both models lead to very similar results. In particular, the most significant variations of the surface coefficient come from the variations of the spin-orbit strength $w_{0}$. On the other hand, the fit to the 


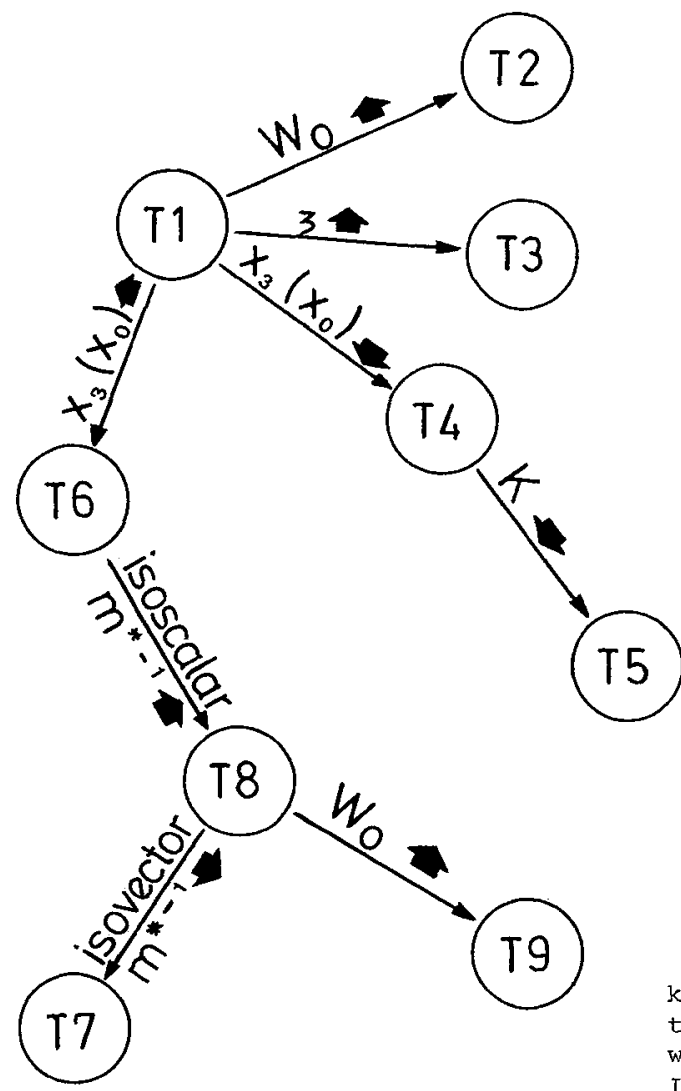

Fig. 4 - Schematic view of the relations between the 9 trial skyrme forces

(see ref. [19] for the definition of these DM coefficients).

b) Several constraints can be obtained by analysing the density distributions. For a reasonable $\mathrm{m}$, the surface diffuseness as well as the compression of the bulk density with respect to nuclear matter mostly depend on the compression modulus $k$ [26]. The value of $\mathrm{K}$ suggested by this analysis ( $\approx 240 \mathrm{MeV}$ ) does not contradict the one deduced from GMR energies [20] $(\approx 220 \mathrm{MeV})$. On the other hand, the neutron skin thickness could be used to fix $J$ and $Q$. The limited accuracy of the available data only defines a possible range $J \approx 29 \pm 2 \mathrm{MeV}$ and $Q \approx 45 \pm 10 \mathrm{MeV}$ (the low $\mathrm{J}$ corresponding to the high Q).

c) It is interesting to note that the small $\left|\nabla\left(\rho_{n}-\rho_{N}\right)\right|^{2}$ term in the skyrme hamiltonian density functional has a significant influence on the amplitude of the shell oscillations of the density in the core region. A force has been given in [21] which allows a good fit to these oscillations. In ETF, this term has no core contribution: it is a good example of the possible differences between ETF and HF.

d) The values of $J$ and $Q$ could also be determined by trying to reproduce fission barrier heights. Table 3 gives ETF barrier heights obtained for 240 Pu. The forces giving a good neutron skin thickness in $208 \mathrm{~Pb}(\mathrm{~T} 6, \mathrm{~T} 7, \mathrm{~T} 8)$ lead to barriers significantly higher than the corresponding DM value $(\approx 3.8 \mathrm{MeV} ;$ the present calculation is limited to the $C$-axis in the $c, h$ parametrisation [22] - However, the spurious rotational energy correction could lead to a significant lowering of these heights. This correction must be included in the microscopic model (i.e. in HF or in the shell correction). As this has not been done usually in the DM + shell correction calculations, the DM barrier height is meaningful only for comparison with a model in which this correction is neglected. Including or neglecting it will lead to different constraints on $J$ and $Q$, and on the related force parameters. The inclusion of the correction seems useful to get an agreement with the values of $J$ and $Q$ obtained from the fit to neutron skins (i.e. quite a low $J$ and quite a high $Q$ ). 


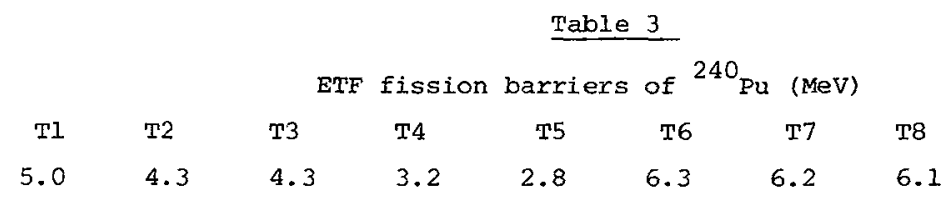

e) The fit of the asymmetry parameters appears as one of the most critical points in the choice of the effective interaction. This fit can also influence other properties, like the giant dipole resonance (GDR) energies. In this case, a third degree of freedom is expressed by the enhancement factor $k$, which is related to the effective masses. Several results [23] suggest $0.6 \leqslant \mathrm{k} \leqslant 0.8$. On the other hand, the GDR energy is not expected to depend very much on $J$ and $Q$, if the force is fitted to the experimental binding energies. This can be shown in the DM where the dependence of $E_{G D R}^{2}$ on $J$ and $Q[24]$ :

$$
E_{G D R}^{2} \sim \varphi(J, Q)=\frac{J}{1+3 J / Q A^{1 / 3}}
$$

is not very different from that of the total (volume + surface) asymmetry energy :

$$
E_{\text {asym. }} \sim \frac{J}{1+9 J / 4 Q A^{1 / 3}}
$$

especially if $J$ is low and $Q$ high, as suggested above. We thus can expect only very rough constraints on $J$ and $Q$ from the analysis of GDR energies. On the other hand, the high values of $K$ suggested by experimental results are only acceptable if $\varphi(J, Q)$ in eq. (15) is quite low. As a general rule, forces leading to the lowest values of $\varphi(J, Q)$ have high $J$ and low $Q$, which seems to contradict the indications given above. In view of the large uncertainties associated with the DM, and with the values of the enhancement factor, we shall limit the present discussion to this remark. Further studies based on less phenomenological models are under way and will be reported elsewhere.

Several results reported in the last section have been calculated by Pr. M. Brack, to whom we are also indebted for very interesting discussions and advice about the strutinsky method and the ETF mode1. We also thank Pr. M. Farine and Dr. D. Berdichevsky for their contribution to specific points of the present study.

\section{References}

I) Vautherin, D. and Brink, D.M., Phys. Rev. C5 (1972) 626

2) Brack, M. , Guet, C., Hăkansson, H.B., submitted to Phys. Rep. (1984) see also : Workshop on Semiclassical methods in nuclear physics, ILL, Grenoble 1981

3) Beiner, M., Flocard, H., Nguyen Van Giai, Quentin, P., Nucl. Phys. A238 (1975) 29

4) Tondeur, F., Pearson, J.M., Farine, M., Nucl. Phys. A394 (1983) 462

5) Vautherin, D., Phys. Rev. C7 (1972) 296

6) Grammaticos, B., voros, A., Ann. Phys. 123 (1979) 359; 129 (1980) 153

7) Strutinsky, V.M., Nucl. Phys. Al22 (1968)/ see also ref. 22

8) Jennings, B.K., Bhaduri, R.K., Brack, M., Nucl. Phys. A253 (1975) 29

9) Brack, M., Quentin, P., Phys. Lett. 56B (1975) 421

10) Strutinsky, V.M., in Physics and Chemistry of Fission, IAEA 1980, p. 475

11) Sobiczewski, A., Gyurkovich, A., Brack, M., Nucl. Phys. A289 (1977) 346

12) Ivanjuk, F., Strutinsky, V.M., Z. Phys. A286 (1978) 291

13) Tondeur, F., Nucl. Phys. A383 (1982) 32

14) Tondeur, F., in Heavy Ion Collisions, Lecture Notes in Physics, Springer verlag 1982 
15) Tondeur, F., Brack, M., Farine, M., Pearson, J.M., to be published in Nucl. Phys. A (1984)

16) Tondeur, F., J. Phys. G5 (1979) 1189

17) Tondeur, F., Nucl. Phys. A311 (1978) 51

18) Bartel, J., Quentin, P., Brack, M., Guet, C., Håkansson, H.B., Nucl. Phys. A386 (1982) 79

19) Myers, W., Swiatecki, W. , Ann. Phys. 55 (1969) 395

20) Treiner, J., Krivine, H., Bohigas, O., Martorell, J., Nucl. Phys. A371 (1981) 253

21) Tondeur, F., Phys. Lett. 123B (1983) 139

22) Brack, M., Damgaard, J., Jensen, A.S., Pauli, H.C., Strutinsky, V.M., Wong, C.Y., Rev. Mod. Phys. 44 (1972) 320

23) Meyer, J., Quentin, P., Brack, M., 7e Session d'Etudes de Physique Nucléaire, Aussois 1983, p. 13-1, LYCEN 8302

24) Myers, W., Swiatecki, W., Kodama, J., El Haick, L., Hilf, E., Phys. Rev. Cl5 (1977) 2032

25) Krivine, H., Treiner, J. Bohigas, O. , Nucl. Phys. A336 (1980) 155

26) Tondeur, F., Berdichevsky,D., in preparation. 